removal, suggesting that the checkpoint is not the formation of a polarized epithelium, but the repulsion of apical surfaces.

Next, the researchers used available genomic data ${ }^{11}$ to gain mechanistic insight into how the exit from naive pluripotency controls cavity formation. This analysis unveiled a potential role for the protein OCT4 in regulating sialomucin expression. When taken together with a recent study ${ }^{12}$ that described the effects of OCT4 deficiency on preimplantation human embryos, the authors' finding suggests an important role for OCT4 in controlling early human development.

Although the expression of sialomucins is essential for cavity formation, the group found that addition of these proteins to naive cells did not, in itself, induce such a transition. This suggests that the delivery of sialomucins to the apical interface might require other proteins, such as the tight-junction protein cingulin, which the researchers showed was produced upon LIF removal. Cingulin has been implicated in the tethering of vesicles to the apical membrane, indicating that it might have a role in cavity formation ${ }^{13}$.

Future work will be needed to identify the cocktail of proteins that is sufficient to trigger naive cells to form a cavity. Moreover, whether the molecular events described in ES cells occur in mouse and human embryos remains to be verified. Understanding how the formation of the amniotic cavity is regulated at the molecular and cellular levels will support future studies of the physical events that control cavity formation, and will lay the foundations for modelling this process ${ }^{2}$.

Altogether, Shahbazi and colleagues' findings allow them to outline a mechanism by which embryos coordinate the exit from pluripotency and the formation of the amniotic cavity. In this scenario, naive cells form a polarized epithelium that is poised for cavitation. Only when cells become primed do their apical interfaces begin to repel one another, allowing fluid to accumulate. This provides evidence that the naive state has a biological role, acting as a checkpoint to ensure that the amniotic cavity forms only when cells are primed. In this way, embryos can keep morphogenesis and differentiation in synchrony.

Julien G. Dumortier and Jean-Léon Maître are in the Unité Génétique et Biologie du Développement, Institut Curie, PSL Research University, CNRS UMR3215, INSERM U934, 75248 Paris, France.

e-mail: jean-leon.maitre@curie.fr

1. Shahbazi, M. N. et al. Nature 552, 239-243 (2017).

2. Maître, J.-L. Biol. Cell 109, 323-338 (2017).

3. Frankenberg, S. R., de Barros, F. R. O., Rossant, J. \& Renfree, M. B. WIREs Dev. Biol. 5, 210-232 (2016).

4. Ying, Q.-L. et al. Nature 453, 519-523 (2008).

5. Shahbazi, M. N. et al. Nature Cell Biol. 18, 700-708 (2016).

6. Deglincerti, A. et al. Nature 533, 251-254 (2016).

7. Bedzhov, I. \& Zernicka-Goetz, M. Cell 156,
1032-1044 (2014)

8. Bryant, D. M. et al. Nature Cell Biol. 12, 1035-1045 (2010).

9. Blasky, A. J., Mangan, A. \& Prekeris, R. Annu. Rev. Cell Dev. Biol. 31, 575-591 (2015)

10.Strilić, B. et al. Curr. Biol. 20, 2003-2009 (2010).
11.Buecker, C. et al. Cell Stem Cell 14, 838-853 (2014)

12. Fogarty, N. M. E. et al. Nature 550, 67-73 (2017).

13. Mangan, A. J. et al. Nature Commun. 7, 12426

(2016).

This article was published online on 29 November 2017.

\title{
ASTROPHYSICS
}

\section{Stellar cannibalism in fits and starts}

\section{Dense stellar remnants called white dwarfs are often found in binary star systems. Satellite observations suggest a previously unknown way in which a white dwarf can draw material from its companion star. SEE LETTER P.210}

\section{THOMAS MARSH}

S even billion years from now ${ }^{1}$, the Sun will evolve into a dense, Earth-sized remnant known as a white dwarf, adding to the billion others that already litter our Galaxy. Many white dwarfs are bound by gravity to other stars, some in such close orbits that they strip mass from their companions. This matter settles onto the white dwarf's surface through a process termed accretion. We thought we knew the main modes of accretion onto white dwarfs, but on page 210 , Scaringi et al. ${ }^{2}$ report that there might be another - one that links white dwarfs to other accreting celestial objects, such as neutron stars.

Accretion is the process by which stars and planets form, and by which the supermassive black holes that lie at the hearts of galaxies are lit up. Accretion onto white dwarfs can cause explosive nuclear-fusion reactions seen most spectacularly in supernovae that can rival entire galaxies in their brightness. In this case, accretion usually occurs in the form of a thin disk of material surrounding the white dwarf in which gas slowly spirals inward on near-circular orbits ${ }^{3}$. As material flows down to the white dwarf, it loses energy, often releasing enough radiation to swamp the combined outputs of the white dwarf and its companion star.

A key property of accretion disks is that they are unstable. They can exist in a cool, dim state for decades, only to switch in a matter of hours to become hot and up to 1,000 times brighter. On shorter timescales (from seconds to hours), disks are animated by a continual jitter in their brightness that never repeats, reflecting the turbulent, dynamic nature of the accretion flow. In both cases, the behaviour was first recognized in white-dwarf systems ${ }^{4}$ before analogous behaviour was identified in related systems, such as those involving young stars ${ }^{5}$ and accreting black holes ${ }^{6}$.

Scaringi and colleagues have discovered a

previously unreported type of behaviour in a white-dwarf system in the constellation Lyra. The system, known as MV Lyrae, normally features a hot, bright accretion disk. However, the system can occasionally fade by more than a factor of 250, sometimes for months at a time. As luck would have it, NASA's Kepler satellite observed MV Lyrae in one of these faint phases, known as low states.

Designed for exoplanet detection, the Kepler satellite has revolutionized the study of stellar variability through its unrivalled stability and because it monitors targets continuously. In this case, it measured MV Lyrae's brightness once per minute for almost four years. Scaringi et al. found that, during a low state, MV Lyrae showed spectacular quasi-regular flaring brightening by as

"The whitedwarf system's brightness was measured once per minute for almost four years." much as a factor of 6 in 30-minute-long bursts that recurred approximately once every 2 hours. This behaviour lasted for days, while the system was in its faint phase. The timescale of these variations implicates the inner part of the accretion disk, close to the white dwarf. So, what causes these bursts?

The authors suggest that we are seeing a form of 'magnetically mediated' accretion (Fig. 1). They propose that a magnetic field, locked to the spinning white dwarf, holds material in the accretion disk at bay until the field is overwhelmed by the build-up of pressure as gas accumulates. Bursts occur as the inner edge of the disk crosses the co-rotation radius - the radius at which the white dwarf's rotation period matches the disk's orbital period $^{7,8}$. This model was developed to explain bursting behaviour from accreting neutron stars ${ }^{7}$, and could match the variability seen in some young stars ${ }^{8}$. However, it has never before been associated with accreting white dwarfs. 


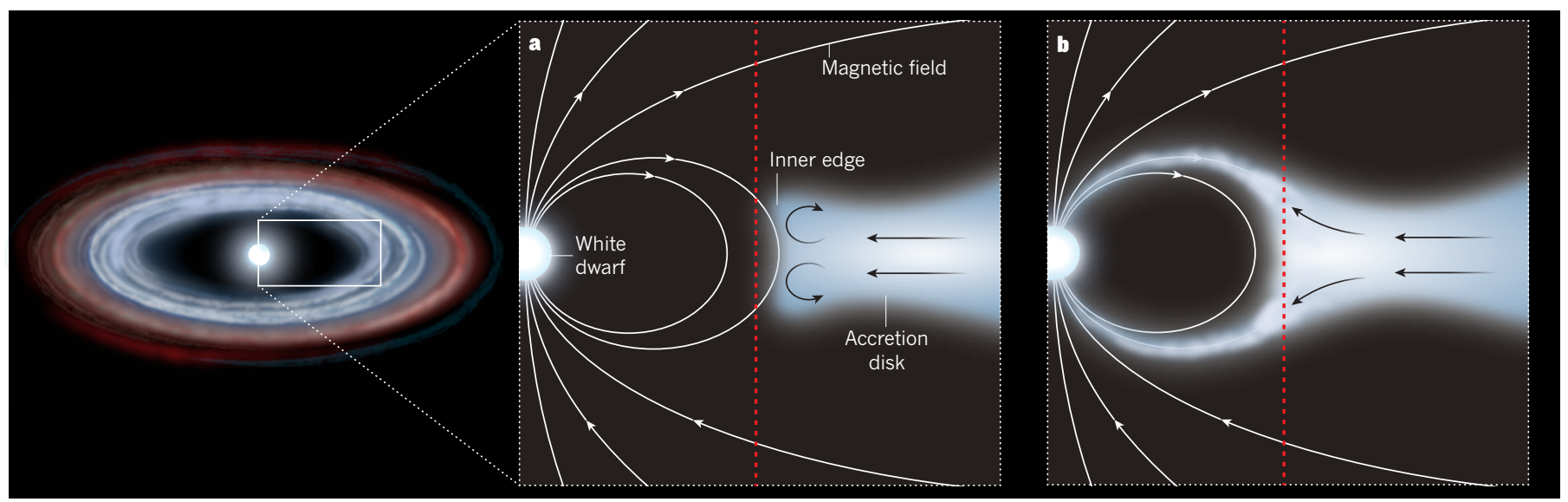

Figure 1 | Magnetically mediated accretion. Stellar remnants called white dwarfs are often surrounded by a ring of gas known as an accretion disk. Scaringi et al. ${ }^{2}$ report a previously unknown way in which this gas can be transferred to the white dwarf's surface. a, A spinning white dwarf produces a magnetic field that holds material in the disk at bay, just outside the co-rotation radius (dotted red line) - the radius at which the white dwarf's rotation period matches the disk's orbital period. Pressure builds as gas accumulates at the inner edge of the disk. The black arrows indicate the flow of gas. b, Eventually, the inner edge of the disk crosses the co-rotation radius, and material travels along magnetic-field lines to the white dwarf's surface. As gas in the disk is depleted, the disk gradually retreats until the magnetic barrier is restored. (Figure adapted from Fig. 3 of ref. 2.)
Although plausible, Scaringi and colleagues' proposal lacks corroborating evidence. In particular, the predicted magnetic field is too weak to be detected easily. Furthermore, it might be so weak that the absence of strictly periodic oscillations in brightness, which often accompany spinning magnetic stars, is no great surprise.

Magnetically mediated accretion is the best model partly because there are no other runners in the race - no alternative model seems to match MV Lyrae's behaviour. Perhaps the main question raised is why similar behaviour has not been reported from any of the other several thousand known accreting white dwarfs. Still, it would be a surprise if accreting white dwarfs, plenty of which are known to be magnetic, did not show this mode of accretion if it occurs in related classes of object.

The discovey of magnetically gated accretion in white-dwarf systems allows plenty of scope for follow-up study. For instance, spectroscopy could tie down the location of the bursts; the magnetic field might be strong enough to generate polarized infrared light, which would confirm the field's presence; and studies of low states in similar objects could establish how common the observed behaviour is. There is much left to be done to investigate this new strand in the web that connects accreting celestial objects. -

\section{A dark side to omega-3 fatty acids}

The molecule 19,20-dihydroxydocosapentaenoic acid, formed by the metabolism of a fatty acid involved in normal brain function, promotes the development of a diabetes-associated form of blindness in a mouse model. SEE LETTER P.248

\section{KEISUKE YANAGIDA \& TIMOTHY HLA}

$\mathrm{D}$ iabetes can have many dangerous complications, including the progressive deterioration of blood vessels in the retina, which can lead to visual impairment. Despite the fact that this condition, called diabetic retinopathy, is a major cause of blindness in middle age, the pathways that mediate its development and progression are poorly understood. Hu et al. ${ }^{1}$ report on page 248 that a derivative of the omega-3 fatty acid docosahexaenoic acid (DHA) has a role in diabetic retinopathy.

Dietary intake of DHA is associated with several health benefits, including normal brain and eye function ${ }^{2}$. Moreover, mice that lack the protein Mfsd2a, which transports DHA across the blood-brain barrier into the brain, develop severe retinal and brain dysfunction $^{3-5}$. Omega-3 fatty acids such as DHA have key functions in cell membranes, where
Thomas Marsh is in the Department of Physics, University of Warwick,

Coventry CV4 7AL, UK.

e-mail:t.r.marsh@warwick.ac.uk

1. Schröder, K.-P. \& Connon Smith, R. Mon. Not. R. Astron. Soc. 386, 155-163 (2008).

2. Scaringi, S., Maccarone, T. J., D'Angelo, C., Knigge, C. \& Groot, P. J. Nature 552, 210-213 (2017)

3. Pringle, J. E. Annu. Rev. Astron. Astrophys. 19, 137-162 (1981).

4. Robinson, E. L. Annu. Rev. Astron. Astrophys. 14, 119-142 (1976).

5. Hartmann, L. \& Kenyon, S. J. Astrophys. J. 299, 462-478 (1985).

6. Mineshige, S. \& Wheeler, J. C. Astrophys. J. $\mathbf{3 4 3 ,}$ 241-253 (1989).

7. Spruit, H. C. \& Taam, R. E. Astrophys. J. 402, 593-604 (1993).

8. D'Angelo, C. R. \& Spruit, H. C. Mon. Not. R. Astron. Soc. 420, 416-429 (2012). they provide membrane fluidity and flexibility, and promote the membranes' role as barriers, allowing the selective transport of molecules into and out of cells ${ }^{6}$.

In addition, omega- 3 fatty acids can have signalling roles. DHA is highly susceptible to oxidation, producing 'lipid mediator' molecules that can regulate cell-cell signalling - for instance, to orchestrate the return to normal conditions following an inflammatory reaction ${ }^{7}$. One class of lipid mediator produced by DHA oxidation is epoxide molecules. These metabolites are rapidly degraded by the enzyme soluble epoxide hydrolase (sEH) to form dihydroxy derivatives such as 19,20-dihydroxydocosapentaenoic acid $(19,20-D H D P)^{8}$. Indeed, $19,20-D H D P$ is the major product of DHA metabolism in the retina?.

Given that DHA and epoxide metabolites are associated with beneficial retinal effects, might sEH play a part in diabetic retinopathy by reducing the levels of these molecules? Hu et al. set out to examine this possibility using a mouse model of the disease. They found that increases in sEH expression in Müller 